\title{
Meigs' Syndromes with Extremelly High CA125 Level Mimicking Advanced Ovarian Cancer
}

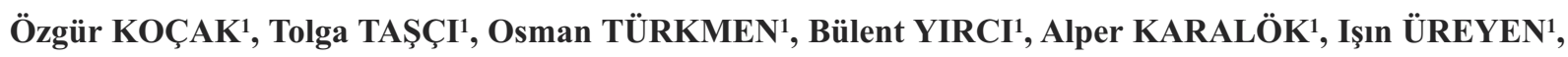 \\ Kerime Nazlı KARAKAYA ${ }^{1}$, Sevgi KOÇ ${ }^{1}$, Taner TURAN ${ }^{1}$, Gökhan TULUNAY1 \\ Ankara, Turkey
}

\begin{abstract}
Meigs' syndrome is defined as pelvic mass with ascites and pleural effusion which can mimic ovarian malignancy. Additionally, elevated serum CA125 level can be seen in Meigs' syndrome. We present 2 cases with solid adnexal mass, ascites and bilateral pleural effusion with high serum CA125 level (2824 $\mathrm{IU} / \mathrm{mL}$ and $1400 \mathrm{IU} / \mathrm{mL}$ ) which were diagnosed as ovarian malignancy preoperatively. In both cases level of CA125 were extremely high for Meigs' syndrome and high level of CA125 in Meigs' Syndrome is unusual. Solid adnexal mass and serous ascites was detected in explorative laparotomy in 2 cases also. Other genital and abdominal structures were normal. In both cases the right salpingo-oophorectomy was performed. In addition, the ovarian fibroma was reported in one case and nonspecific for other case during frozen/section examination. Contralateral salpingo-oophorectomy and total hysterectomy was performed because of the age of patients in one case and non- diagnostic frozen section examination in the other case. Final pathology confirmed the diagnosis of ovarian fibroma in first case and thecoma in the second one. Despite the suggestion of ovarian malignancy in preoperative period, the frozen/section examination is the mainstay in the management of adnexal masses.
\end{abstract}

Keywords: Meigs' syndrome, Ovarian mass, Ascites, High level CA 125

Gynecol Obstet Reprod Med 2016;22:42-44

\section{Introduction}

Although the ovarian cancer is the sixth most common cancer in women worldwide it is the most common reason of death among all causes of the most gynecologic malignancy in Western Europe and North America. ${ }^{1}$ Symptoms associated with ovarian cancer are often nonspecific, and the majority of patients continue to present with advanced stage of disease..$^{2-3}$ Ovarian cancer mostly seen in postmenopausal women, with a median age at diagnosis of 60 years. ${ }^{1}$ More than one third of ovarian cancer patients present with malignant ascites at diagnosis. ${ }^{4}$ Ascites is also present in $10-15 \%$ of Meigs' syndrome. ${ }^{5}$ In postmenopausal women elevated serum CA125 (cancer antigen 125 , carcinoma antigen 125 , or carbohydrate antigen 125) with solid adnexal masses, ascites, and pleural effusion are highly suggestive for ovarian malignant tumor. However ovarian malignancy diagnosis is made by histopathology with frozen/section examination during surgery or after surgery.

\footnotetext{
${ }^{I}$ Division of Gynecologic Oncology Etlik Zübeyde Hanım Women's Health Teaching and Research Hospital, Ankara

Address of Correspondence: Özgür Koçak Etlik Zubeyde Hanim Women's Health

Teaching and Research Hospital Division of Gynecologic Oncology, Etlik, Keçiören, Ankara dr.ozgur@hotmail.com

Submitted for Publication: $\quad$ 27.08.2014

Accepted for Publication: $\quad 24.09 .2014$
}

Minority of these patients may have a benign condition known as Meigs' syndrome. This benign condition can be treated only by removal of the pelvic tumor.

Meigs' syndrome is firstly described in 1937 by Meigs and Cass that presented report describing 7 cases with following findings: ascites, ovarian tumor and pleural effusion resolved by removal of the tumor. ${ }^{6}$ Classification of Meigs' syndrome was restricted to bening and solid ovarian tumors by Meigs in 1954.7 The term Pseudo Meigs' syndrome is used in the case if other tumors such as primary or metastatic tumors of uterin or fallopian tubes are found. Although the high level of CA125 is unusual in Meigs' syndrome in the literature Walker et al. reported the case with the highest level of CA125 which was more than $5000 \mathrm{IU} / \mathrm{mL} .{ }^{8}$ The first case with high level of CA125 in Meigs' syndrome was reported by Jones OW; Surwit EA in $1989 .{ }^{9}$

We report 2 cases of Meigs' syndrome caused by right ovarian fibroma/thecoma with extremely elevated serum CA125 level in postmenopausal women.

\section{Case 1}

A 60 years old woman with right pleural effusion, massive ascites and a pelvic mass in the right adnexal region was initially diagnosed with advanced stage ovarian cancer and admitted to the gynecologic oncology department on January 
2014. Ultrasound and computed tomography confirmed a $12 \times 10 \mathrm{~cm}$ right adnexal mass, bilateral pleural effusion and ascites. Serum Ca125 level was $2824 \mathrm{IU} / \mathrm{mL}$. Explorative laparotomy was performed. $4500 \mathrm{cc}$ ascites and $12 \mathrm{~cm}$ right adnexal mass was observed. Other pelvic and abdominal structures were normal. Right salpingo-oophorectomy was performed. Ovarian fibroma was reported by frozen/section examination (and then contralateral oophorectomy and total hysterectomy was performed because of the age of the patient. Final pathology confirmed the diagnosis of ovarian fibroma.

\section{Case 2}

A 58 years old woman with right pleural effusion, massive ascites and a pelvic mass in the right adnexal region was referred to our hospital from periphery on May 2014 with diagnosis of an advanced stage of ovarian cancer. Ultrasound examination was performed and revealed $214 \times 129 \mathrm{~mm}$ right adnexal mass with ascites and in the inferior part of the mass has a 93x72 mm solid part. Serum Ca125 level was $1400 \mathrm{IU} / \mathrm{mL}$. Explorative laparotomy was performed and $3000 \mathrm{cc}$ ascites and $15 \mathrm{~cm}$ right adnexal mass was observed without adhesion. The mass was ruptured during operation so the mass size was smaller than the preoperative ultrasonografic size. The mass was well circumscribed with a thick wall and has solid component (Figure 1,2). Other pelvic and abdominal structures were normal. Right salpingo-oophorectomy was performed and specimen sent to frozen section examination. Frozen section examination showed that the resected mass was highly suggestive for benign and that paraffin block should be performed in

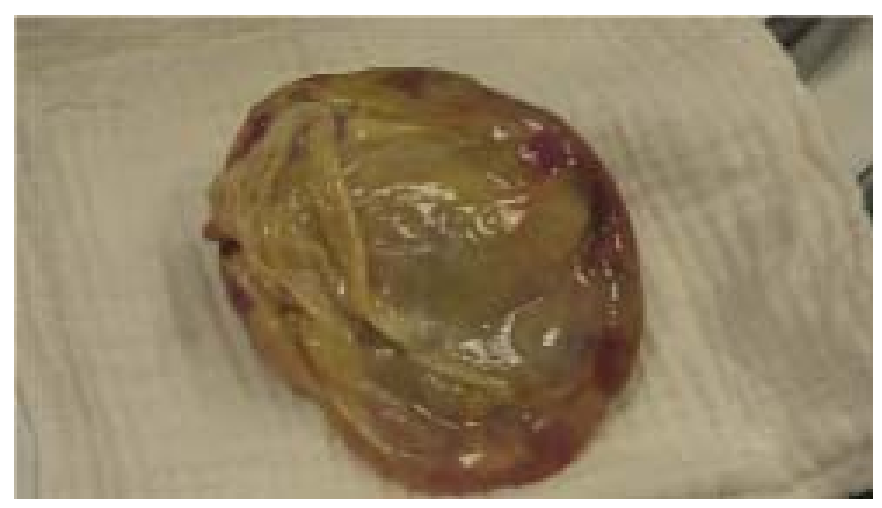

Figure 1: The adnexal mass was ruptured during operation, well circumscribed with a thick wall and has solid component

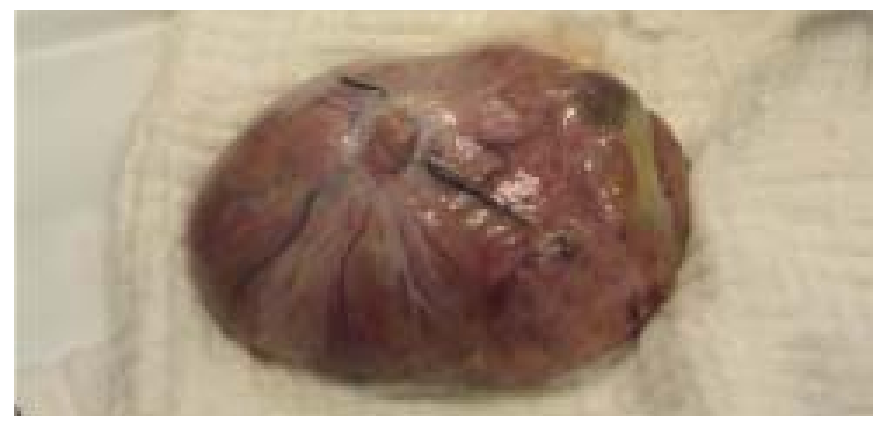

Figure 2: The solid part of the mass order to make final decision. Therefore contralateral oophorectomy and total hysterectomy was performed. Other pelvic and abdominal structures were found to be normal. Final pathology confirmed the diagnosis of ovarian fibro- thecoma.

\section{Discussion}

In 1937, firstly Meigs and Cass reported a series of 7 patients with ascites and pleural effusions and benign ovarian fibromas. All these patients were treated only by removal of the benign ovarian masses. ${ }^{6}$ Meigs' syndrome is defined by a triad that pleural effusion, ascites and benign ovarian masses. It is typically more common in postmenopausal women with an average age of about 50 years. Many theories have been postulated to explain the origins of the ascitic and pleural fluids in Meigs' syndrome. Ascites occur most probably due to transudative mechanism from the tumor surface that exceeds the resorption capacity of peritoneum. ${ }^{10}$ Meigs et al. explained that tumor itself makes the pressure on the lymphatics and causes the escape of fluid through the surface of lymphatics . ${ }^{7}$ The reason for the pleural effusions are thought arise direct passage of ascitic fluid from defects in the diaphragm, and the diaphragm defects mostly seen in right side of the diaphragm and so the right pleural effusions in Meigs' syndrome. ${ }^{7}$ The pleural and peritoneal exudates are identical biochemical and cellular compositions. ${ }^{11}$

CA-125 also known as mucin 16 or MUC16 is a protein that in humans is encoded by the MUC16 gene. ${ }^{12}$ The serum CA125 concentration may be elevated in a lots of conditions such as advanced endometrial and cervical adenocarcinoma and benign conditions as pelvic inflammatory disease, uterine fibroids, pregnancy, spontaneous abortion with chromosomal abnormality, Meigs' syndrome, and especially endometriosis. ${ }^{13}$ Serum CA-125 levels are also increased with peritoneal, pleural, and pericardial inflammation or irritation, and there is evidence that peritoneal mesothelial cells are even more potent than ovarian cancer cells in producing CA-125. ${ }^{14}$ When considering possible explanation of the elevated serum CA-125 level all the conditions above must be kept in mind. Our cases showed extremely elevated serum CA-125 level with a laboratory finding of Meigs' syndrome. Associated pathology of the pelvis and the interval between diagnosis of the diseases and surgery can also play a role in the mechanisms of developing elevated serum CA-125 levels. Therefore, late recognition of ovarian fibroma/thecoma complicated with Meigs' syndrome in our cases could thus explain the unusual finding of extremely high level of CA125 that was described in previous reports.

In patients with an unexplained pleural effusion or ascites, the Meigs' syndrome should be considered. Before the definition of the Meigs' syndrome, patients with ascites, pleural effusion and ovarian masses thought to be inoperable due to the clinical pictures of a malignant ovarian tumor with pleural metastases and effusion. Therefore, by presenting these 2 
cases we want to draw an attention to the fact that Meigs' syndrome should be included in the differential diagnosis of women with the triad of the syndrome. Frozen section examination is the main part of the treatment patients with triad of pelvic mass, ascites and pleural effusion. It is obvious that more aggressive surgery should be done to the patient whose frozen section examination refers a malignancy. The treatment of the Meigs' syndrome is done only by the removal of the adnexal masses. In our first case the surgeons completed the treatment of patient by removing the mass but they preferred to do contralateral oophorectomy and total hysterectomy because the patient was 60 years old. In second case the frozen section fails to fully distinguish benign and malign condition surgeons decided to do contralateral oophorectomy and total hysterectomy. In both cases the major determinant factor in surgery was frozen section examination.

\section{Reference}

1. Parkin DM, Bray F, Ferlay J, Pisani P. Global cancer statistics, 2002; CA Cancer J Clin 2005;55:74-108.

2. Goff BA, Mandel L, Muntz HG, Melancon CH. Ovarian carcinoma diagnosis. Cancer 2000; 89:2068-75

3. Goff BA, Mandel LS, Dresden CW, et al. Development of an ovarian cancer symptom index: possibilities for earlier detection. Cancer 2007;109:221-7.

4. Kipps E, Tan DS, Kaye SB. Meeting the challenge of ascites in ovarian cancer: new avenues for therapy and research. Nat Rev Cancer 2013;13(4):273-82.

5. Loué VA, Gbary E, Koui S, Akpa B, Kouassi A. Bilateral ovarian fibrothecoma associated with ascites, bilateral pleural effusion, and marked elevated serum ca-125. Case Rep Obstet Gynecol 2013;2013:189072

6. Meigs JV, Cass JW. Fibroma of the ovary with ascites and hydrothorax: a report of 7 cases. Am J Obstet Gynecol 1937;33:249-67

7. Meigs J. Fibroma of the ovary with ascites and hydrothorax-Meigs' syndrome. Am J Obstet Gynecol 1954;67: 962-87

8. Walker JL, Manetta A, Mannel RS, Liao SY. Cellular fibroma masquerading as ovarian carcinoma. Obstet Gynecol 1990;76:530e1

9. Jones OW $3^{\text {rd }}$, Surwit EA. Meigs syndrome and elevated CA125. Obstet Gynecol 1989;73:520-1

10. Samanth KK, Black III WC. Benign ovarian stromal tumors associated with free peritoneal fluid. Am J Obstet Gynecol 1970;107:538-45

11. Hoffman MS. Peritoneal tuberculosis, large ovarian thecoma and an elevated serum CA 125 level mimicking ovarian cancer. J Florida Med Ass 1989;76(4):388-9

12. Yin BW, Lloyd KO. Molecular cloning of the CA125 ovarian cancer antigen: identification as a new mucin, MUC16. J Biol Chem 2001;276(29):27371-5.

13. Jacobs I, Bast RCJ. The CA 125 tumor-associated antigen: a review of the literature. Hum Reprod 1989;4:1-12.

14. Zeimet AG, Marth C, Offner FA. Human peritoneal mesothelial cells are more potent than ovarian cancer cells in producing tumor marker CA-125. Gynecol Oncol 1996; 62:384-9. 